\title{
Water reactor fuel performance code PROFESS and its application for predicting the behaviour of the fuel elements of D-Com blind problem
}

\author{
D N SAH, D VENKATESH and E RAMADASAN \\ Radiometallurgy Division, Bhabha Atomic Research Centre, Trombay, Bombay 400085 , \\ India

\begin{abstract}
This paper gives a brief description of the sub-models of important phenomena treated in computer code PROFESS and presents some results of PROFESS calculations for the
\end{abstract} \\ fuel elements of D-Com blind problem.
}

Keywords. Computer modelling; computer code; nuclear fuel element; water reactor; code verification; fuel performance; irradiation effects; irradiation behaviour.

\section{Introduction}

The first version of computer code PROFESs (performance analysis of rod-type oxide fuel elements under steady state) was completed in the beginning of 1983. To check the predictive capability of the code and its sub-models, it was necessary to simulate a few documented irradiation experiments and to compare the predictions with the experimental results. At that time, a code exercise was being organized as part of an IAEA co-ordinated research programme on development of computer models (D-Com) to investigate the predictive capability of the existing codes. A detailed data package on an irradiation experiment was made available to the participants of the exercise and it was known as D-Com blind problem. The predictions of the different codes participating in this exercise were to be compared with the actual experimental findings, which had been held secret till calculations of all codes had been submitted. This provided a good opportunity to check the predictive capability of PROFESS by participating in this exercise. This paper briefly describes the models of some important phenomena treated in the code and gives some of the results obtained on the fuel elements of D-Com blind problem.

\section{Computer code PRofess}

Computer code PROFESs is applicable to rod-type fuel elements used in water-cooled nuclear reactors. A typical water reactor fuel rod consists of a number of sintered $\mathrm{UO}_{2}$ or $\mathrm{UO}_{2}-\mathrm{PuO}_{2}$ fuel pellets stacked inside a zircaloy cladding tube which is sealed at both ends.

The general features of Profess have been described elsewhere (Sah et al 1983; Sah and Venkatesh 1984). The flow chart of calculation followed in PROFESS is given in figures 1 and 2. The models incorporated in this code are listed in table 1. The general 


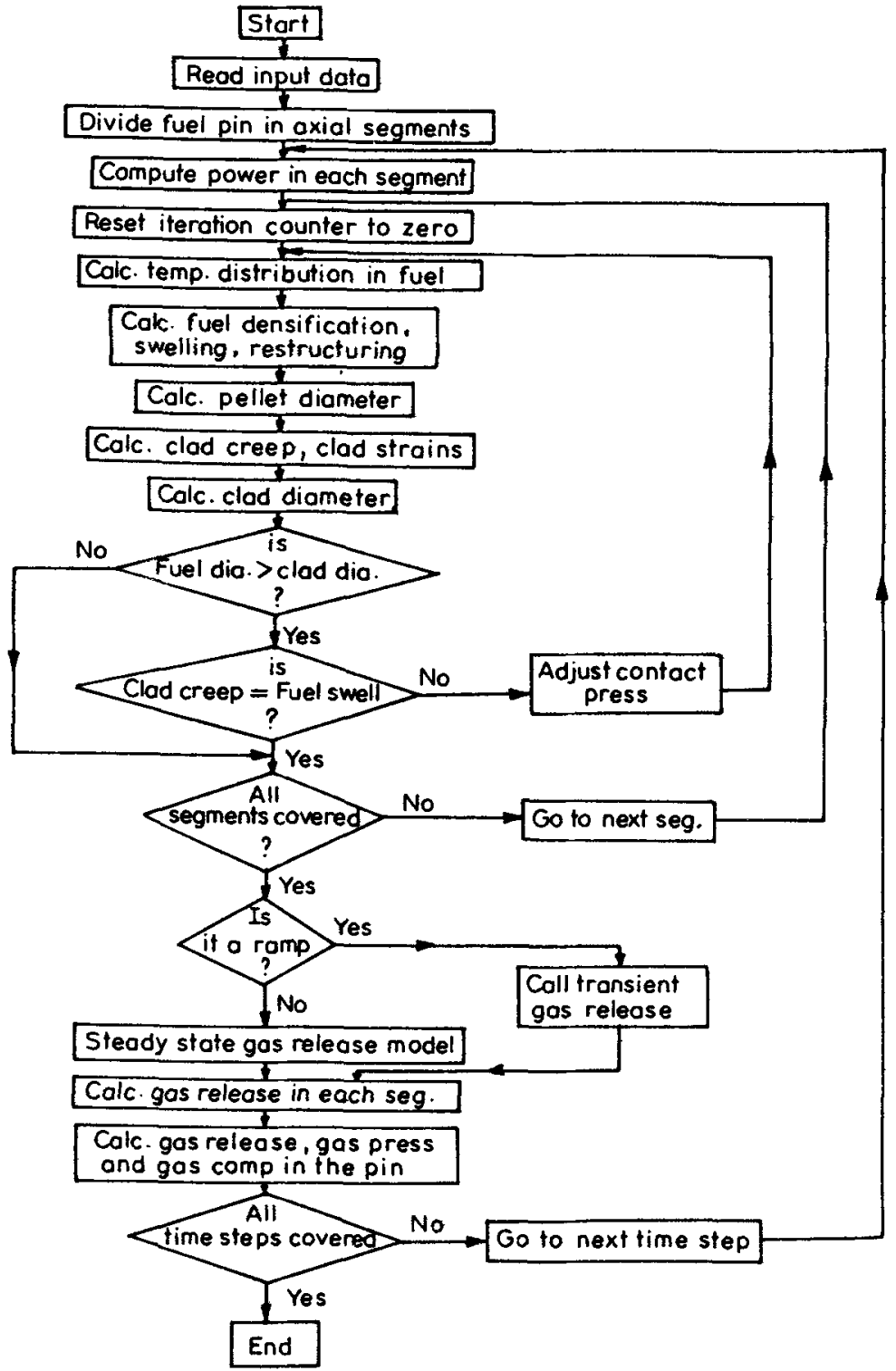

Figure 1. Flow chart of the calculations followed in PROFESS.

method of thermal and mechanical analysis of fuel element is described elsewhere (Roy and Sah 1985). Models of important phenomena treated in PROFESs are briefly described below.

\subsection{Gap conductance model}

The heat flow through the fuel clad gap/interface is considered to take place by thermal conduction and radiation. The gap conductance is evaluated by the Ross-Stout model 


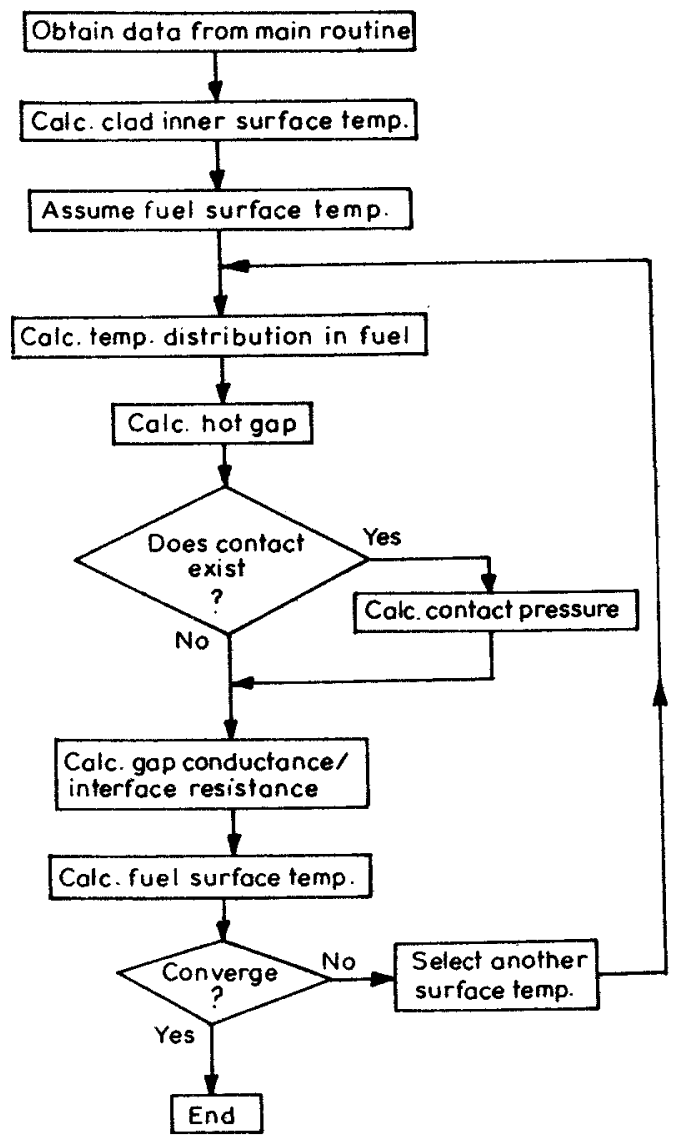

Figure 2. Flow chart of the calculation of radial temperature distribution in the fuel pin cross-section.

Table 1. Models in computer code PROFESS

Analytical models

Cladding surface temperature

Temperature drop across cladding

Temperature drop across fuel clad-gap

Radial temperature distribution in fuel

Elastic deformation of cladding

Void volume

Internal gas pressure

Fission gas generation

Gap gas dilution

Plenum temperature

Neutron flux depression
Material properties models

Thermal expansion of $\mathrm{UO}_{2}$

Thermal expansion of zircaloy

Thermal conductivity of $\mathrm{UO}_{2}$

Thermal conductivity of zircaloy

Thermal conductivity of gases, $\mathrm{He}, \mathrm{Xe}$

Thermal conductivity of gas mixture

Jump distance of gases

Yield strength of zircaloy

Poisson's ratio of zircaloy

Meyer's hardness of zircaloy

Elastic modulus of zircaloy

\section{Phenomenological models}

Fuel clad gap conductance

Solid and gaseous fission product swelling

In pile fuel densification

Fission gas release (steady state)

Fission gas release (transient)

Equiaxed grain growth

Columnar grain growth

Fuel relocation

Zircaloy in-pile creep 
(Ross and Stout 1962) modified to account for the effect of fission gas, gas pressure and pellet eccentricity.

When the fuel-clad gap is open, the heat transfer coefficient in the gap is given by

$$
h_{\text {gap }}=h_{\text {cond }}+h_{\text {rad }}
$$

where

$$
\begin{aligned}
h_{\text {cond }} & =K_{\text {gas }} /\left(X_{\text {gap }}+\delta+g_{f}+g_{c}\right), \\
h_{\text {rad }} & =\left(4 \sigma T^{3}\right) /\left\{\left[\left(1 / E_{f}\right)+\left(1 / E_{c}\right)\right]\right\}-1,
\end{aligned}
$$

$K_{\text {gas }}=$ thermal conductivity of the gas, $\delta=$ sum of the surface roughness of fuel and cladding, $g_{f} g_{c}=$ jump distances of the gas in the fuel and in the cladding, $X_{\text {gap }}=$ fuelclad gap, $\sigma=$ Stefan-Boltzmann constant, $E_{f} E_{c}=$ emissivity of the fuel and the cladding and $T=$ temperature in the fuel clad gap. When the fuel-clad gap is closed i.e. the fuel and cladding are in contact with each other, the heat transfer co-efficient through the interface $\left(h_{\text {cont }}\right)$ is given by

$$
h_{\mathrm{cont}}=\frac{K_{\mathrm{gas}}}{\delta+g_{c}+g_{f}}+C \frac{2 K_{f} K_{c}}{\left(K_{f}+K_{c}\right)} \cdot \frac{P_{c}}{\delta^{1 / 2} H},
$$

where $K_{f}, K_{c}$ are thermal conductivities of fuel and cladding, $P_{c}$ is the contact pressure between fuel and cladding, $H$ is the Meyer's hardness of cladding and $C$ is a constant.

In the gap conductance model used in PROFEss it is assumed that when the pellet is placed eccentrically, its effect can be evaluated by assuming that even in the open gap condition a certain fraction of fuel surface is in pressureless contact with the cladding. This contact area fraction depends upon the existing gap and is defined as follows:

$$
C A=1-\left(\frac{\text { hot gap }}{\text { cold gap-Ao }}\right)^{1.5 \varepsilon^{2}},
$$

where $\varepsilon$ denotes the extent of eccentricity and has a value between 0 and 1 , for a fully concentric pellet, $\varepsilon$ is zero resulting in a contact area fraction of zero and $A_{0}$ is a constant. The method of solution in the gap conductance model used in PROFESs is similar to the method used in COMETHE code (Hoppe et al 1982).

\subsection{In-pile fuel densification}

The irradiation-induced densification of uranium dioxide fuel is considered to be a function of the initial fuel density, the amount of unstable porosity in the fuel and the fuel burn-up. The model in PROFESS considers that the initial unstable pores disappear exponentially with the progress of fuel irradiation. The volume change in the fuel due to in-pile densification is given by the following correlation (Pedersen 1978),

$$
(\Delta V / V)_{\mathrm{dens}}=\left(P_{0}-P_{s}\right)\left[\exp \left(\frac{-\mathrm{BU}}{500}\right)-1\right],
$$

where $(\Delta V / V)_{\text {dens }}$ is the volumetric change in the fuel, $P_{0}$ is the initial porosity fraction, $P_{s}$ is the fraction of stable porosity in the fuel and BU is fuel burn-up. The densification is considered isotropic. 


\subsection{Solid and gaseous fission product swelling}

The fission product swelling model incorporated in PROFEss considers free swelling of fuel when the fuel clad gap is open and a restrained swelling occurs when the fuel and cladding are in pressure contact with each other. The swelling correlations have been derived from the experimental data reported in literature (Zimmermann 1979; Hilbert et al 1971). Below $900^{\circ} \mathrm{C}$ free swelling is a linear function of fuel burn-up and above this temperature the swelling is considered as a function of fuel burn-up and fuel temperature both. The restrained swelling is linearly related to fuel burn-up and it is taken as $1 \%(\Delta V / V)$ per at. $\%$ burn-up.

\subsection{Fission gas release}

There are five optional fission gas release models (Sah et al 1984) in profess for calculating the steady state fission gas release from fuel. The transient fission gas release is estimated by a model given below (Pati and Ritterbusch 1980).

$$
F=1-\exp \left(-m t^{n}\right)
$$

where $F=$ fraction of pre-transient gas released during the transient, $t=$ time at transient (seconds), $m=$ temperature dependent variable (given by $2.22 \times 10^{-7} \times$ $\left.(T-1000)^{2}\right), T=$ fuel temperature ${ }^{\circ} \mathrm{C}$ and $n=0.25$. In the above model the gas release occurs only if the fuel temperature exceeds $1000^{\circ} \mathrm{C}$. The generation of fission gas during transient is ignored and the release occurs from the retained gas available in the fuel. At fuel burn-up higher than $20,000 \mathrm{MWD} / \mathrm{MTU}$ a correction factor is used to account for burn-up enhancement of the steady state release.

\subsection{Fuel restructuring}

Two important microstructural changes in fuel e.g. equiaxed grain growth and columnar grain growth have been modelled in PROFESS. The correlations used are given below.

Equiaxed grain growth is represented by the following correlation

$$
D^{2}=D_{0}^{2}+2.92 \times 10^{-8}\left[\exp -\frac{2.67 \times 10^{5}}{8.314 T}\right] \cdot t,
$$

where $D_{0}$ is the initial grain size and $D$ the final grain size. The grains are allowed to grow to a limiting size which is given by

$$
D_{\max }=2.23 \times 10^{-3} \exp (-7620 / T) \text {. }
$$

The columnar grain growth is represented by a time temperature relationship given by $t=t_{0} \exp (A / T+273)$, where $t$ is the time required to form columnar grains at a temperature $T\left({ }^{\circ} \mathrm{C}\right), A$ and $t_{0}$ are constants.

\subsection{Cladding creep}

The creep rate of zircaloy cladding is considered a function of cladding stress, fast neutron flux $(>1 \mathrm{MeV})$ and the cladding temperature. The correlation used in PROFESS is given below (Wood and Watkins 1971). 


$$
\dot{\varepsilon}=A \exp \left(-\frac{14000}{R T}\right) \phi^{0.85} \sin (\sinh (0-165 \sigma))
$$

where $A$ is a constant, $\phi$ is the fast neutron flux, $\sigma$ is cladding stress $\mathrm{kg} / \mathrm{mm}^{2}, T$ is cladding temperature $\left({ }^{\circ} \mathrm{C}\right.$ ) and $R$ is the gas constant.

\section{Results of PROFESS calculation of D-Com blind problem}

\subsection{D-Com blind problem}

The D-Com blind problem refers to an irradiation experiment in which three (AG11-8, AG11-9 and AG11-10) pre-pressurised mini-fuel elements were irradiated together to a fuel burn-up of about $32000 \mathrm{MWD} / \mathrm{MTU}$ at fairly low heat ratings. Two of these fuel elements were subjected to a power ramp (referred as bump test) at the end of life. The third fuel element (AG11-8) had been taken out of reactor prior to the bump test. The design of the fuel elements, the axial power profile in the fuel assembly and the power history of irradiation is shown in figure 3. Misfeldt (1982) compiled the details of this irradiation experiment and the data package used for the code calculations.

\subsection{PROFESS calculations}

PROFESS was used to calculate the following parameters as a function of irradiation time (i) fuel centre temperature (ii) gap conductance (iii) fission gas release in the fuel element (iv) fuel restructuring and (v) fuel element diameter. The detailed results of the calculation have been reported elsewhere (Sah et al 1984). Some important results related to the gap conductance, fission gas release and fuel restructuring are presented below.

3.2a Fuel-clad gap conductance: The variation of gap conductance as a function of irradiation time in the three fuel elements at their mid-stack location is shown in figure 4. A gradual increase in the gap conductance is observed in all the three fuel elements in the beginning, followed by an abrupt increase to a comparatively high value at about $10,000 \mathrm{hrs}$. The sudden increase in the gap conductance indicates the occurrence of fuel-cladding contact in the fuel elements. However it is seen that the value of gap conductance in the three elements is different from each other, even though the fuel clad gap has closed in all the three. This difference is because of the different amount of fission gas released in the three fuel elements (see $\$ 3.2 \mathrm{~b}$ ). Since fission gases, $\mathrm{Xe}$ and $\mathrm{Kr}$, have very low thermal conductivity a higher gas release in a fuel element results in a lower value of gap conductance. The results presented in this figure, when compared with the experimentally measured fission gas release in the three fuel elements, indicates that PROFESs is able to simulate the gap conductance behaviour very well.

3.2b Fission gas release: The amount of gas release calculated by PROFESs in the three fuel elements is given in table 2 along with the experimentally measured values. There is satisfactory agreement between the predicted and measured gas release in the fuel element AG 11-8. However an underprediction of about $30 \%$ is noted in the bump tested elements AG11-9 and AG11-10. Comparison of the PROFESS predictions with the 


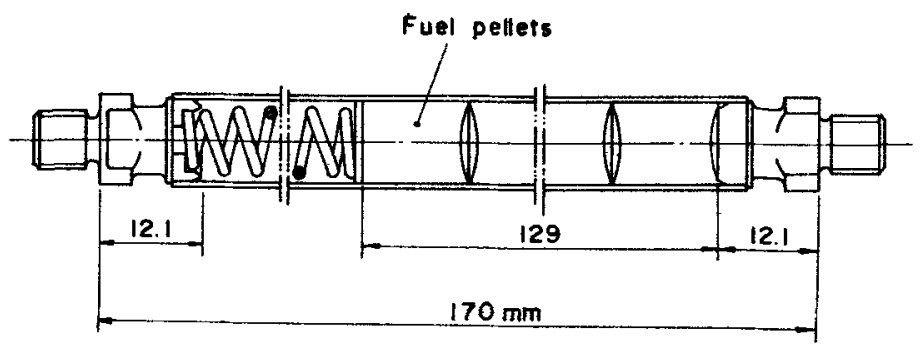

(a)

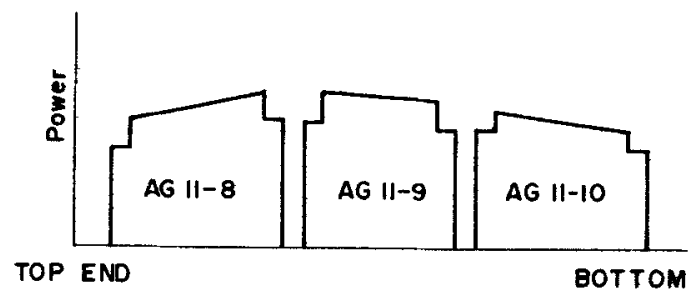

(b)

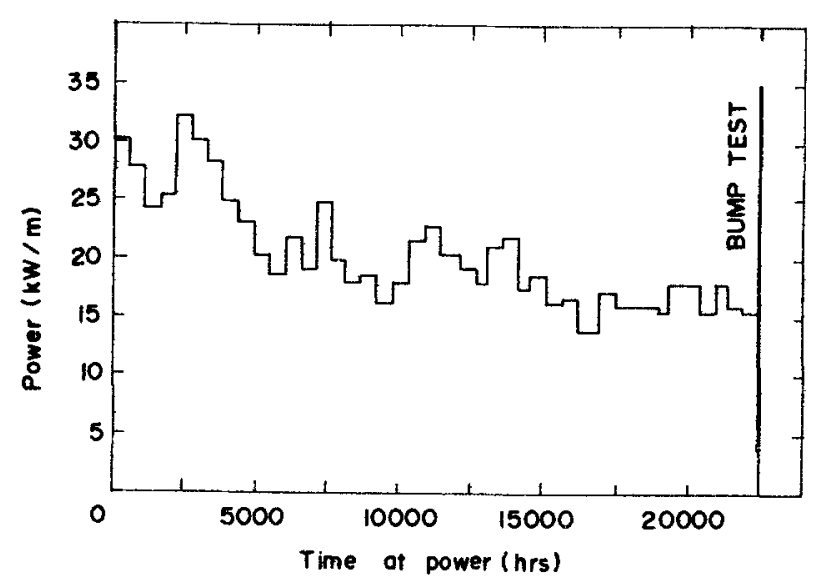

(c)

Figure 3. The design and the irradiation history of the fuel elements of D-Com blind problem; (a) fuel pin schematic design (b) power profile in the fuel pin assembly and (c) irradiation power history.

prediction of some other codes (figure 5) revealed that most of the codes tended to underpredict the gas release in these fuel elements. This indicates that the model of transient gas release adopted by the codes are presently inadequate for accurate prediction. 


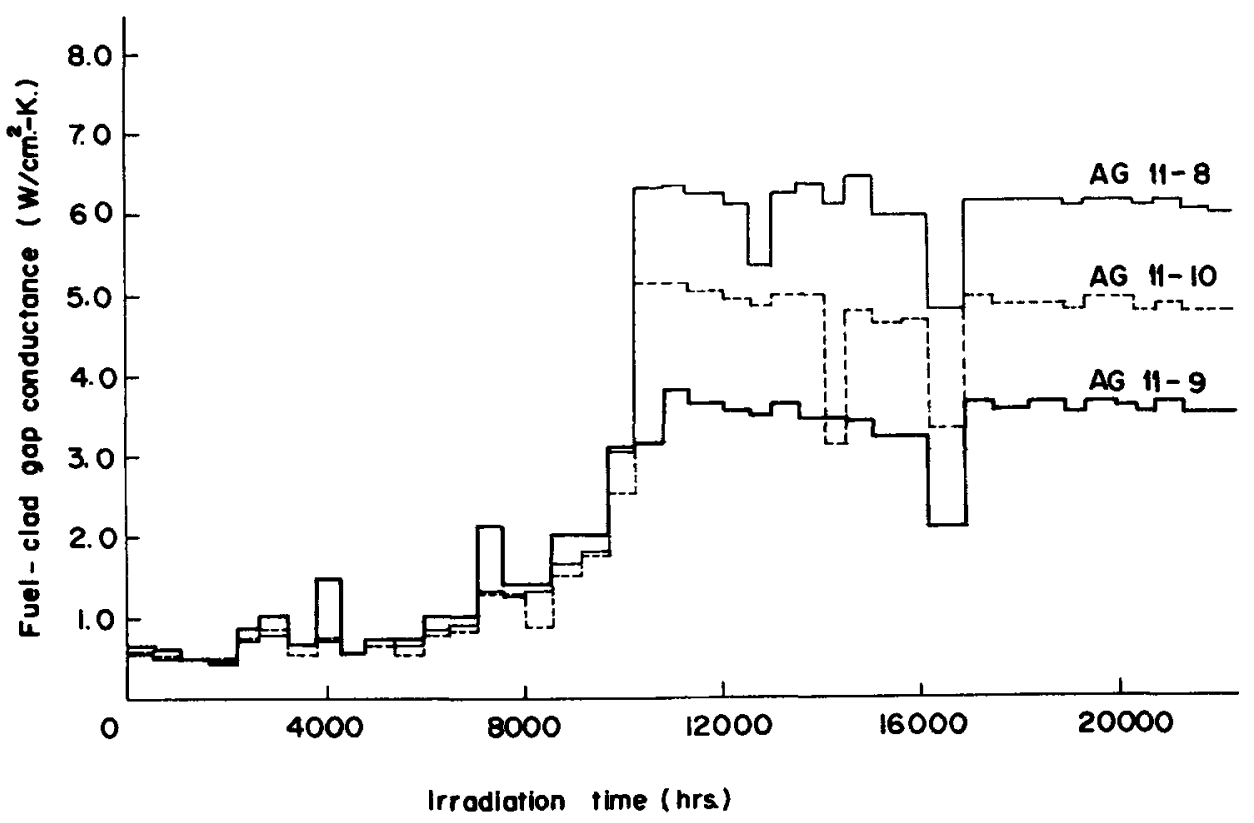

Figure 4. Fuel-clad gap conductance at the mid-stack location in the three fuel elements, as a function of irradiation time.

Table 2. Comparison of the predicted and the measured values of fission gas release in the three fuel elements of D-Com blind problem

\begin{tabular}{lcc}
\hline & \multicolumn{2}{c}{ Fission gas release (cc STP) } \\
\cline { 2 - 3 } & $\begin{array}{c}\text { PROFEss } \\
\text { Fued element }\end{array}$ & $\begin{array}{c}\text { Experimentally } \\
\text { measured }\end{array}$ \\
\hline AG 11-8 & 1.25 & 1.5 \\
AG 11-9 & 9.0 & $12 \cdot 5$ \\
AG 11-10 & 2.81 & 4.5 \\
\hline
\end{tabular}

The gas release behaviour (as a function of irradiation time) calculated by PROFESS in AG 11-9 and AG 11-10 is shown in figure 6. It is interesting to note that in AG 11-9, the major amount (about two third of the total) of gas release occurred during the bump testing. This trend was in agreement with the conclusion arrived from the experiment (Misfeldt 1983).

3.2c Fuel restructuring: The calculations indicated that no grain growth occurred in the fuel element AG 11-8. This prediction is in agreement with the experimental finding. PROFESS prediction of the extent of grain growth at fuel centre and the size of the equiaxed grain growth region in the fuel element AG 11-9 was found to be in very good 


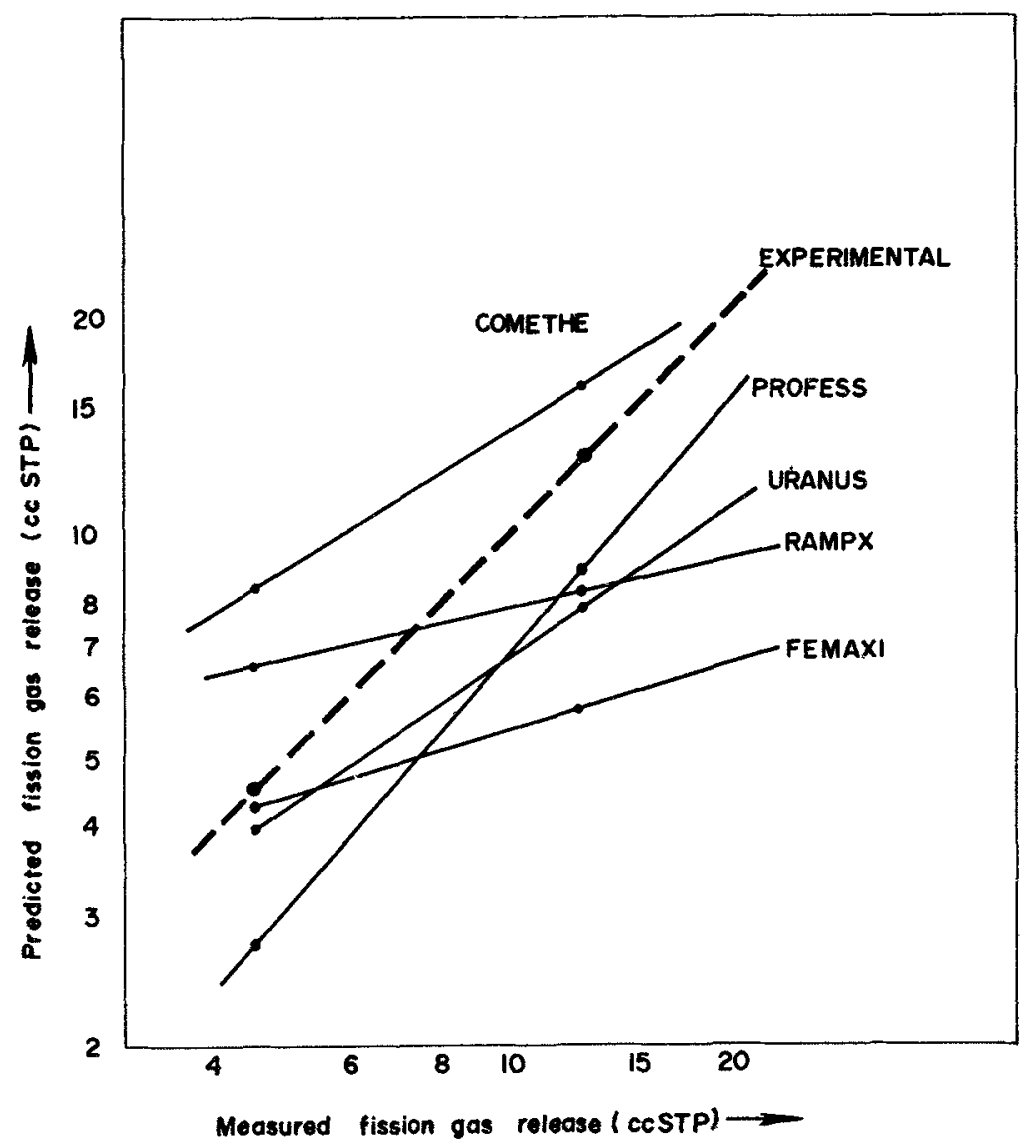

Figure 5. Comparison of the measured fission gas release in AG 11-9 and AG 11-10, with the prediction of some codes.

Table 3. Comparison of the grain growth prediction with experimental observation at the mid-stack location in the fuel elements

\begin{tabular}{lccccc}
\hline & \multicolumn{2}{c}{$\begin{array}{c}\text { Fuel grain size } \\
\text { at the centre } \\
(\mu \mathrm{m})\end{array}$} & & \multicolumn{2}{c}{$\begin{array}{c}\text { Size of grain } \\
\text { growth region } \\
\text { (fuel-fractional radius) }\end{array}$} \\
\cline { 2 - 5 } \cline { 5 - 6 } Fuel element & Predicted & Observed & Predicted & Observed \\
\hline AG 11-8 & No grain growth & No grain growth & 0.00 & 0.00 \\
AG 11-9 & $32 \cdot 4$ & 30 & & 0.57 & 0.50 \\
AG 11-10 & $24 \cdot 2$ & Not measured & 0.51 & Not measured \\
\hline
\end{tabular}

agreement with metallographic observations (table 3). The calculations also revealed that the grain growth in AG 11-9 occurred during the initial period of irradiation (figure 7). 


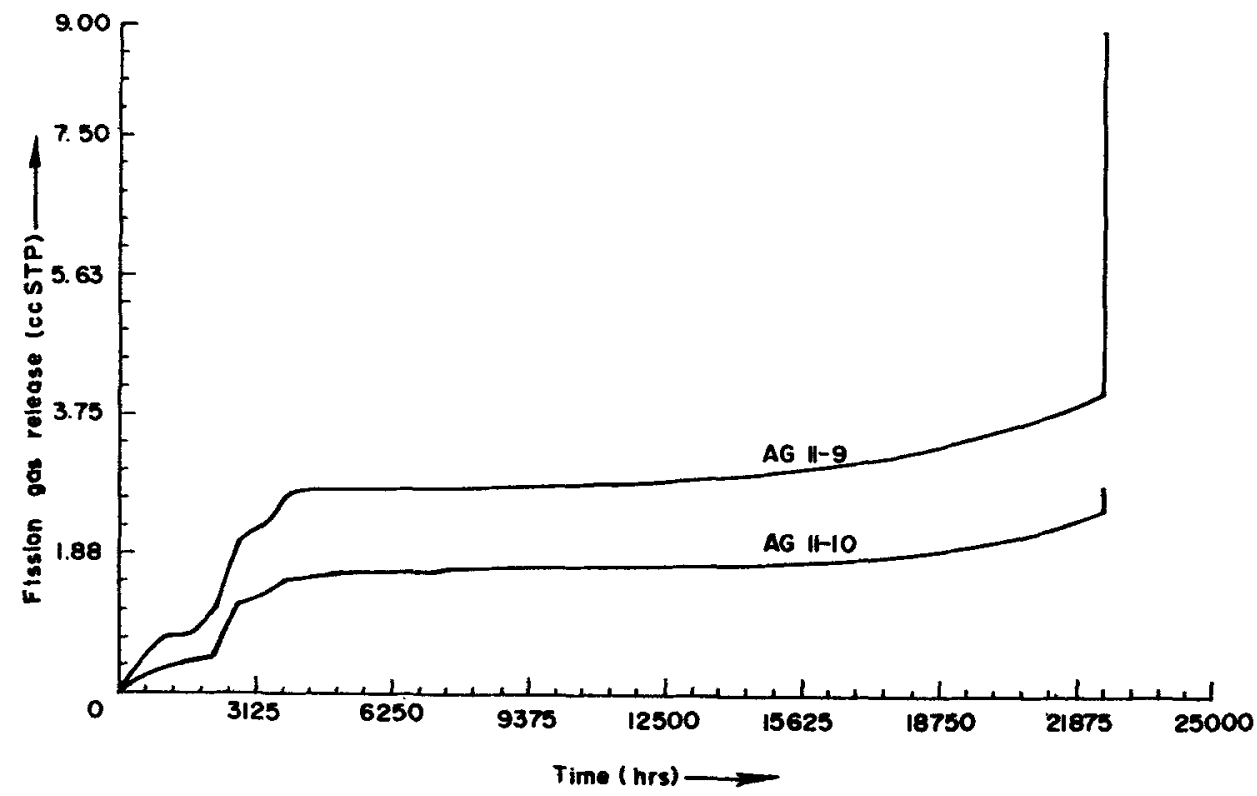

Figure 6. Predicted fission gas release in the fuel elements AG 11-9 and AG 11-10 as a function of irradiation time.

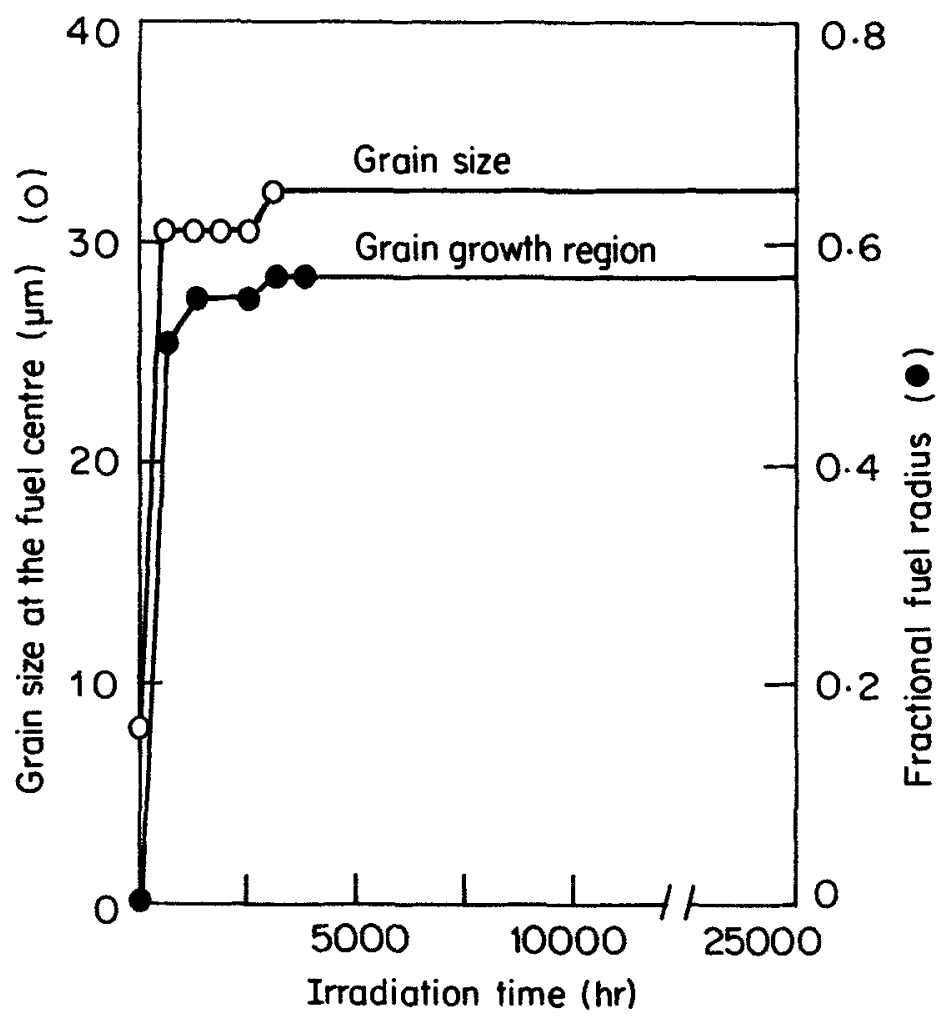

Figure 7. Predicted grain size at the fuel centre and the size of the grain growth region in the pellet cross-section for fuel elements AG 11-9, as a function of irradiation. 


\section{Conclasion}

Computer code PROFESS was successfully used to calculate the performance parameters of the three fuel elements of an irradiation experiment commonly referred to as D-Com blind problem. The prediction of the fission gas release in the fuel elements and the fuel restructuring was found to be in reasonable agreement with experimental observations. The fuel-clad gap conductance model gives good simulation of the effect of fission gases and clearly revealed the occurrence of fuel-clad gap closure in the fuel elements during irradiation. It was noted, however, that there is a tendency for under-prediction of gas release during transient and better models may be required to represent the gas release in the fuel elements during transient conditions.

\section{Acknowledgement}

The authors wish to express their sincere thanks to Shri K S Sivaramakrishnan of PIE Section and Shri P R Roy, of Metallurgy Group for their keen interest in this work.

\section{References}

Hilbert R F, Storhok V W, Chubb W and Keller D C 1971 J. Nucl. Mater. 3826

Hoppe N, Billaus M and Vliet J U 1982 Belgonuclair Report COMETHE III-L, Report No. BN $8201 /-04$

Misfeldt I 1982 Data for The D-Com blind problem on fission gas release, Report B-528, Metallurgy Dept., RIS $\emptyset$ National Laboratory, Denmark

Misfeldt I 1983 OECD-NEA-CSNI/I AEA Specialists meeting on Water Reactor Fuel Safety and Fission Gas release in off normal and Accident Condition, RIS $\emptyset$ National Laboratory, Denmark

Pati S R and Ritterbusch S E 1980 Fission gas release during anticipated transient; IAEA Specialists Meeting on Water Reactor Fuel Element Performances Modelling, Blackpool, UK

Pedersen N K 1978 Proc. I AEA Specialists Meeting on Water Reactor Fuel Performance Computer Modelling, Blackpool, UK

Ross A M and Stout R L 1962 Heat Transfer Coefficient between $\mathrm{UO}_{2}$ and Zircaloy-2, Report AECL 1552.

Roy P R and Sah D N 1985 Pramana (J. Phys.) 24397

Sah D N, Venkatesh D and Sivaramakrishnan K S 1983 Proc. Symp. on Irradiation Effects in Solids (Bombay: BARC) p. 203

Sah D N, Venkatesh D and Ramadasan E 1984 IAEA Specialists Meeting on Water Reactor Fuel Element Performance Modelling, Bowness on Windermere UK (to be published).

Wood D S and Watkins B 1971 J. Nucl. Mater. 41327

Zimmermann H 1979 IAEA Specialists Meeting on Behaviour of defected zirconium alloy clad ceramic fuel in water cooled reactors, Chalk River, Canada p. 147 